Dept. of Poultry Diseases

Animal Health Research Institute, Zagazig

\title{
EFFECT OF TOLTRAZURIL AND AMPROLIUM PLUS IN BROILER CHICKENS INFECTED WITH COCCIDIOSIS
}

(With 5 Tables)

\author{
By \\ H.H. ALLAM; H.A.A. BAYOUMIE; \\ SEHAM M. H. MALHAT* and I.A.A. MOHAMED** \\ * Dept. of Chemistry, Animal Health Research Institute, Zagazig \\ ** Dept. of Avian and Rabbit Med. Fac. of Vet. Med. Zag. Univ.
}

(Received at 12/6/2008)

تأثير التولترازوريل والأمبرول بلس فى بدارى دجاج التسمين المصابة

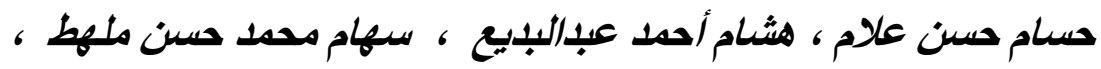

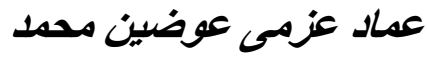

أجريت هذه الدراسة لتقييم الاستعمال المنز امن للتولترازوريل و الأمبرول بلس سوياً فى علاج

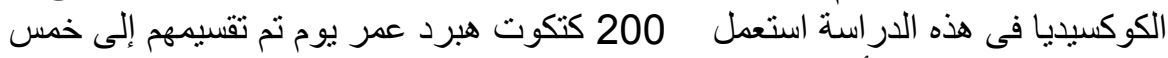

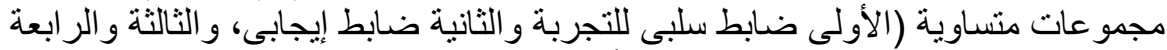

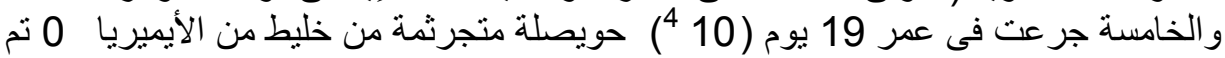

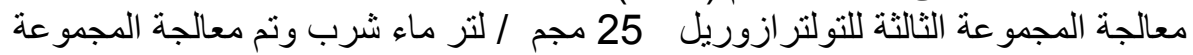

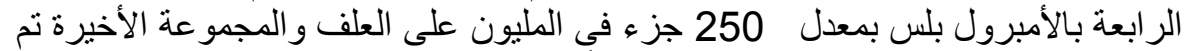

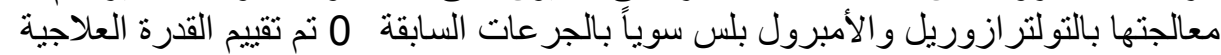

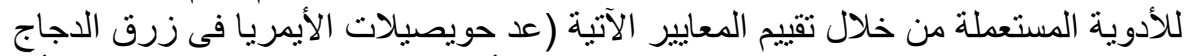

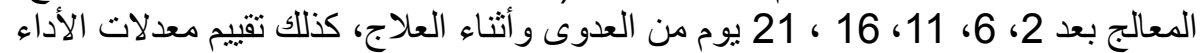

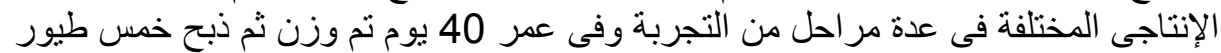

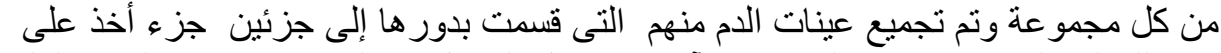

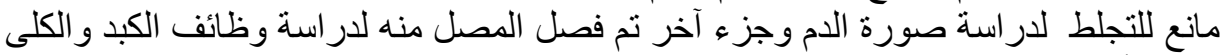

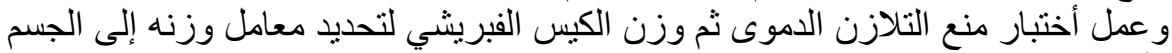

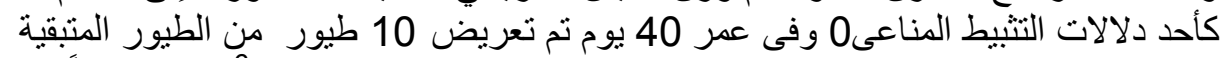

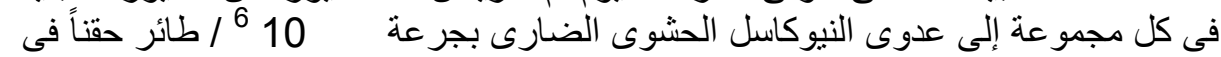
العضل لتقييم التنبيط المناعى أيضا0 من النتائج النى تم التوصل بلت إليها يمكن القول أن أستخدام 


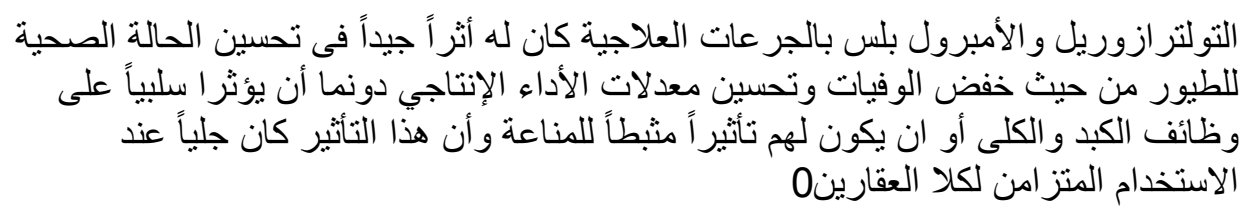

\section{SUMMARY}

The present study was performed to evaluate the concurrent use of toltrazuril and amprolium plus in treating avian coccidiosis. Two hundred one day old Hubbard broiler chicks kept in wire floor batteries under hygienic measures were used. On day $19^{\text {th }}$ of age chicks were aliquated into five groups, 40 each $\left(1^{\text {st }}\right.$ group served as negative control, $2^{\text {nd }}$ group served as positive control, $3^{\text {rd }}$ group was infected and treated with toltrazuril $25 \mathrm{mg} / \mathrm{L}$ of drinking water, $4^{\text {th }}$ group was infected and treated with Amprolium plus $250 \mathrm{ppm}$ in ration and the $5^{\text {th }}$ group was infected and concurrently treated with toltrazuril $25 \mathrm{mg} / \mathrm{L}$ and amprolium plus $250 \mathrm{ppm}$ in ration. Treatment usually started 3 days post infection, chicken in all groups were weighted at 20,27, 34 and 41 day of age. Body weight gain, feed consumption, feed conversion, mortality, lesion score was determined. Oocyst count was performed 2, 6, 11, 16 and 21 days PI. On the $40^{\text {th }}$ day of age, 5 birds from each group were weighted, slaughtered and had bursal body weight index determined. Blood samples from slaughtered birds were divided into two portions, one for serum preparations for serology and clinical chemistry parameters, while the other portion was collected on EDTA for hematological study. 10 of the remaining birds from each group were subjected for virulent Newcastle diseases challenge. Results obtained showed that tolrazuril and amprolium plus administration individually at the therapeutic doses had valuable effect in the treatment of avian coccidiosis and simultaneous administration of both drugs had more potent curative effect and less effect on liver and kidney function parameters, meanwhile non of the used drugs proved immunosuppressive.

Key words: Coccidiosis, broiler chickens, toltrazuril, amprolium plus 


\section{INTRODUCTION}

Coccidiosis is a disease of universal importance in poultry production, it causes intestinal damage with resulting interruption of feeding, digestive processes, nutrient absorption and dehydration, beside blood loss and increased susceptibility to other diseases agents (Mc Dougald 2003).

Eimeria spp. causing coccidiosis in chickens are ubiquatous, and it has been said that, the only limit to their distribution is the distribution of their host (Jordan and Pattison 1996).

Several key factors are responsible for the epidemiology of coccidiosis and its ubiquitous nature. These are, the long persistence of Oocyst in the environment, the short prepatent period and the high biotic potential of the parasite. There is no maternally derived immunity for early protection and that immunity is acquired by infection and maintained by continual infection., and the attak with one spp. of parasite does not confer full protection against another spp. Coccidial oocyst can tolerate most disinfectants and only ammonia and methylbromide gas can effectively kill oocysts but they are highly noxious for poultry and working personnel thus they can not be used during disease outbreak. The moisture, oxygen and warmth required for sporulation are similar to those required for sound poultry breeding (Jordan, 1990; Jordan and Pattioon 1996; Alexander, 2002; McDougald 2003).

Control of coccidiosis relies principally on drugs Alexander (2002), Amprolium and toltrazuril are the usual drugs used during outbreak of coccidiosis, but development of tolerance for drugs after exposure in a serious limitation for the effectiveness of drug (McDougald, 2003).

This work, was planned to evaluate the simultaneous use of toltrazuril (Tol) and amprolium (Amp) plus in treating avian coccidiosis through the parasitological clinicopathological studies as well as changes in body weight, oocyst output, lesion score, mortality rate and possible immunosuppression in broiler chickens. 


\section{MATERIALS and METHODS}

\section{Materials}

\section{Chickens:}

Two hundred day old Hubbard broiler chicks, obtained from Cairo poultry company were used in the present study.

\section{Drugs:}

\section{Toltrazuril}

Toltrazuril (Bayer) 2.5\% was used at a dose of $25 \mathrm{mg} / \mathrm{L}$ of drinking water (Vertommen and Peek 1990).

\section{Amprolium plus:}

Amprolium plus (merial) each gm contains $250 \mathrm{mg}$ amporlium HCL and $16 \mathrm{mg}$ ethopabate, the drug was used at a dose of $250 \mathrm{ppm}$.

\section{Ration:}

High energy, high protein ration "super starter ration" Cairo poultry company was used ad libitum.

\section{Mixed Eimeria sporulated oocysts:}

Intestinal contents from field cases suffering coccidiosis submitted to the animal health res. Lab. Zagazig was preserved in $4 \%$ potassium dichromate and aerated with aquarium pump to promote sporulation as described by McDougald (2003).

\section{Viruses:}

\section{Live virus vaccines:}

$\mathrm{NDV}\left(\mathrm{HB}_{1}\right.$, and LaSota) intervet, IBDV (D-78) intervet were used according to their manufactures.

\section{Challenge NDV:}

A VVNDV with a titer of $10^{7.7} \mathrm{EID}_{50} / 0.1 \mathrm{ml}$ Bayoumie et al. (2006) was used.

\section{Chicken embryos:}

Nine day old chicken embryos from native balady breeding flock reared in Sharkia was used for virus propagation and antigen preparation.

\section{Viral antigens:}

ND Hemagglutination antigen:

Allantoic fluid from chicken embryos inoculated with Losata NDV was used as hemagglutinating antigen for $\mathrm{HI}$ - test (Allan et al. 1978). 


\section{ND precipitating antigen}

The CAM of chicken embryos inoculated with Lasota NDV was prepared as described by Bayoumie (1997) and used as ND precipitating antigen.

ND precipitating antiserum:

Serum was prepared earlier (Bayoumie et al., 2006).

\section{Methods:}

\section{Experimental design:}

Table 1 summarizes the distribution of experimental birds and experimental design.

Table 1: Outlines the performed experimental procedure

\begin{tabular}{|c|c|c|c|c|c|c|c|}
\hline \multirow{2}{*}{ 苛 } & \multirow{2}{*}{ 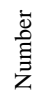 } & \multirow[t]{2}{*}{ Experimental groups } & \multicolumn{2}{|c|}{ Preventive vaccination } & \multirow{2}{*}{$\begin{array}{l}\text { Inoculation } \\
\text { schedule }\end{array}$} & \multicolumn{2}{|c|}{ Sampling \& experimental procedure } \\
\hline & & & Age & Vaccine & & Calender & Procedure \\
\hline A & 40 & $\begin{array}{c}\text { Negative control non infected } \\
\text { no treated } \\
\text { (but vaccinated) }\end{array}$ & $7 \mathrm{D}$ & HB, eye drops & $\begin{array}{c}\text { Group } \\
\text { B,C,D,E } \\
\text { Were crop }\end{array}$ & $\begin{array}{c}\text { At } 20,27,34 \\
\& 41 \text { days } \\
\text { of age }\end{array}$ & B.W., WG, FCR \\
\hline B & 40 & $\begin{array}{l}\text { Positive control infected } \\
\text { non treated }\end{array}$ & $14 \mathrm{D}$ & $\begin{array}{l}\text { IBDV eye } \\
\text { drops }\end{array}$ & $\begin{array}{l}\text { inoculated } \\
\text { with } 10^{4}\end{array}$ & $\begin{array}{c}2,6,11,16 \& \\
21 \text { day PI }\end{array}$ & Oocyst output count \\
\hline $\mathrm{C}$ & 40 & $\begin{array}{lcr}\text { Infected, treated } & \text { with } \\
\text { toltrazuril } & 25 & \mathrm{mg} / \mathrm{L} \\
\text { drinking water } & \end{array}$ & $18 \mathrm{D}$ & Lasota D.W. & $\begin{array}{c}\text { sporulated } \\
\text { Eimeria } \\
\text { oocyst /bird }\end{array}$ & $\begin{array}{c}\text { At } 40 \text { day of } \\
\text { age }\end{array}$ & $\begin{array}{l}\text {-Blood collection on EDTA } \\
\text { for hematology } \\
\text {-Serum collection for AST, }\end{array}$ \\
\hline $\mathrm{D}$ & 40 & $\begin{array}{llr}\text { Infected , } & \text { treated } & \text { with } \\
\text { amprolium } & 250 \mathrm{ppm} / \quad \mathrm{kg} \\
\text { feed } & & \end{array}$ & & & & & $\begin{array}{l}\text { ALT, ALP, GGT uric acid } \\
\text { creatinine \& HI } \\
\text {-B.B. index }\end{array}$ \\
\hline $\mathrm{E}$ & 40 & $\begin{array}{l}\text { Infected, treated with (Tol+ } \\
\text { Amp) simultaneously }\end{array}$ & & & & & $\begin{array}{l}-\mathrm{VVNDV} \text { challenge } \\
10^{6} \mathrm{VP} / \mathrm{IM}\end{array}$ \\
\hline
\end{tabular}

$\mathrm{BW}=$ Body weight, $\mathrm{WG}=$ weight gain, $\mathrm{FCR}=$ feed conversion ratio, $\mathrm{VP}=$ virus particle, $\mathrm{IM}=\mathrm{Intramuscular}$, AST=Asptrate immunotrasnferase , ALT=alanine aminotransferase, ALP = Alkaline phosphatase , GGT= gama glutamy transferase, $\mathrm{HI}=$ haemaggultination inhibition, $\mathrm{BB}$ index= bursal body weight index , VVNDV= velogenic viscerotropic Newcastle viruses

\section{Experimental coccidial infection:}

On $19^{\text {th }}$ day of age group B, C, D, E were crop inoculated with 1 x $10^{4}$ mixed eimeria oocysts. (Karim and Tress, 1990).

\section{Coccidal lesion score:}

It was performed according to Johnson and Reid (1970).

\section{Productive performance parameters:}

Body weight (BW), weight gain (WG) and feed conversion rate (FCR) were performed for all chicken groups at 20, 27, 34 and 41 day of age. 


\section{Oocyst output count:}

At 2, 6, 11, 16 and 21 day PI oocyst output was counted using McMaster Technique as described Johnson and Reid (1970).

\section{Hematological and clinicochemical parameters:}

At 40 day of age blood was collected on EDTA for determination of hemogram and total leukocytic count according to Coles (1986), another part of the blood sample was used to obtain serum for determination of serum transaminases (AST - ALT) (Reitman and Frankel 1957), Gamma glutamyltransferase (GGT) (Szaz, 1969), serum alkaline phosphatase Tietz (1986), serum uric acid (Caraway, 1957), creatinine (Husdan and Rapoport 1968).

\section{Hemagglutination and hemagglutination inhibition:}

They were performed as described by Villegas (1991).

\section{Evaluation of immunosuppression:}

Immunsuppression due to IBDV vaccination and treatment with Toltrazuril and amprolium was tested by serologic and challenge results, beside bursal body weight index (Lucio \& Hitchner 1979).

Statistical analysis:

Statistical analysis was done after Snedecor and Cochran (1967).

\section{RESULTS}

Results of the present work are illustrated in Tables (2-5)

Table 2: Shows mortality, lesion score and oocyst count in the different experimental groups 2, 6, 11, 16 and 21 day post infection.

\begin{tabular}{|c|c|c|c|c|c|c|c|c|c|}
\hline \multirow{2}{*}{$\begin{array}{l}\text { Experimental } \\
\text { groups }\end{array}$} & \multirow{2}{*}{$\begin{array}{c}\text { Total } \\
\text { number }\end{array}$} & \multicolumn{2}{|c|}{ Mortality } & \multirow{2}{*}{$\begin{array}{l}\text { Lesion } \\
\text { score }\end{array}$} & \multicolumn{5}{|c|}{ Oocyst count in gm faeces $\times 10^{3}$} \\
\hline & & Number & $\%$ & & 2 D.PI & 6D.PI & 11D.PI & 16D.PI & 21D.PI \\
\hline $\begin{array}{l}\text { a. Non infected non } \\
\text { treated }\end{array}$ & 40 & 0 & 0 & 0 & 0 & 0 & 0 & 0 & 0 \\
\hline $\begin{array}{l}\text { b. Infected non } \\
\text { treated }\end{array}$ & 40 & 4 & 10 & 4 & $\begin{array}{l}2.22 \pm \\
0.08\end{array}$ & $\begin{array}{c}4.17 \pm \\
0.18\end{array}$ & $\begin{array}{l}5.49 \pm \\
1.15\end{array}$ & $\begin{array}{l}7.27 \pm \\
0.73\end{array}$ & $\begin{array}{l}7.52 \pm \\
1.36\end{array}$ \\
\hline $\begin{array}{l}\text { c. Infected treated } \\
\text { with (Tol) }\end{array}$ & 40 & 0 & 0 & 2 & $\begin{array}{c}0.52 \pm \\
0.09 * * *\end{array}$ & $\begin{array}{c}0.36 \pm \\
0.16^{* * *}\end{array}$ & $\begin{array}{l}1.74 \pm \\
0.3 * * *\end{array}$ & $\begin{array}{l}2.71 \pm \\
0.78 * * *\end{array}$ & $\begin{array}{l}2.07 \pm \\
0.59 * * *\end{array}$ \\
\hline $\begin{array}{l}\text { d. Infected treated } \\
\text { with (Amp) }\end{array}$ & 40 & 1 & 2.5 & 2 & $\begin{array}{c}0.52 \pm \\
0.11 * * *\end{array}$ & $\begin{array}{c}0.33 \pm \\
0.32 * * *\end{array}$ & $\begin{array}{l}1.82 \pm \\
0.3 * * *\end{array}$ & $\begin{array}{l}2.05 \pm \\
0.3 * * *\end{array}$ & $\begin{array}{r}2.26 \pm \\
0.18 * * *\end{array}$ \\
\hline $\begin{array}{l}\text { e. Infected treated } \\
\text { with (Tol \& } \\
\text { Amp) }\end{array}$ & 40 & 0 & 0 & 1 & $\begin{array}{l}0.61 \pm \\
0.3 * * *\end{array}$ & $\begin{array}{c}0.24 \pm \\
0.19 * * *\end{array}$ & $\begin{array}{l}1.44 \pm \\
0.2 * * *\end{array}$ & $\begin{array}{c}1.99 \pm \\
0.16^{* * * *}\end{array}$ & $\begin{array}{l}1.31 \pm \\
0.17^{* * * *}\end{array}$ \\
\hline
\end{tabular}

$* * *=$ High significant $\mathrm{P}<0.001$ 
Assiut Vet. Med. J. Vol. 54 No. 118 July 2008

Table 3: The hematological findings, liver and kidney functions in the different experimental groups PI at 40 days of age.

\begin{tabular}{|c|c|c|c|c|c|c|c|c|c|c|c|c|c|}
\hline \multirow{2}{*}{ 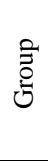 } & \multicolumn{7}{|c|}{ Hematological findings } & \multicolumn{4}{|c|}{ Liver functions } & \multicolumn{2}{|c|}{ Kidney function } \\
\hline & $\begin{array}{c}\text { RBCs } x \\
10^{6} / \mathrm{ml}\end{array}$ & $\mathrm{Hb} \%$ & PCV & $\begin{array}{c}\text { WBCs x } \\
10^{3} / \mathrm{ml}\end{array}$ & $\mathrm{MCV}$ & $\mathrm{MCH}$ & $\mathrm{MCHC}$ & $\begin{array}{l}\text { ALT } \\
\mu / \mathrm{ml}\end{array}$ & $\begin{array}{l}\text { AST } \\
\mu / \mathrm{ml}\end{array}$ & ALP & GGT & $\begin{array}{c}\text { Uric } \\
\text { acid } \\
\mathrm{mg} / \mathrm{dl}\end{array}$ & $\begin{array}{c}\text { Creatinine } \\
\mathrm{Mg} / \mathrm{dl}\end{array}$ \\
\hline $\mathrm{a}$ & $\begin{array}{c}3.04 \pm \\
0.2\end{array}$ & $\begin{array}{l}8.97 \pm \\
0.74\end{array}$ & $\begin{array}{c}30.04 \pm \\
1.21\end{array}$ & $\begin{array}{c}31.82 \pm \\
0.92\end{array}$ & 98.8 & 29.5 & 29.8 & $\begin{array}{c}28.19 \pm \\
1.23\end{array}$ & $\begin{array}{c}30.76 \pm \\
1.87\end{array}$ & $\begin{array}{c}23.16 \pm \\
1.31\end{array}$ & $\begin{array}{c}21.08 \pm \\
1.73\end{array}$ & $\begin{array}{c}8.03 \pm \\
0.94\end{array}$ & $\begin{array}{l}1.02 \pm \\
0.08\end{array}$ \\
\hline $\mathrm{b}$ & $\begin{array}{l}2.1 \pm \\
0.34\end{array}$ & $\begin{array}{c}7.06 \pm \\
0.17\end{array}$ & $\begin{array}{c}27.17 \pm \\
0.22\end{array}$ & $\begin{array}{c}34.27 \pm \\
0.26\end{array}$ & 129.3 & 33.61 & 25.0 & $\begin{array}{l}35.32 \pm \\
0.83 * *\end{array}$ & $\begin{array}{l}39.28 \pm \\
0.94 * *\end{array}$ & $\begin{array}{l}29.34 \pm \\
0.87 * *\end{array}$ & $\begin{array}{l}25.26 \pm \\
0.26 * *\end{array}$ & $\begin{array}{l}10.73 \pm \\
0.36 \text { ** }\end{array}$ & $\begin{array}{l}1.48 \pm \\
0.1^{*}\end{array}$ \\
\hline $\mathrm{c}$ & $\begin{array}{l}2.92 \pm \\
0.48\end{array}$ & $\begin{array}{l}8.03 \pm \\
0.32\end{array}$ & $\begin{array}{c}28.62 \pm \\
0.28\end{array}$ & $\begin{array}{c}32.18 \pm \\
0.61\end{array}$ & 98 & 27.5 & 28 & $\begin{array}{c}31.21 \pm \\
0.31\end{array}$ & $\begin{array}{c}34.18 \pm \\
1.38\end{array}$ & $\begin{array}{c}25.92 \pm \\
0.38\end{array}$ & $\begin{array}{c}23.61 \pm \\
0.21\end{array}$ & $\begin{array}{c}9.73 \pm \\
0.14\end{array}$ & $\begin{array}{l}1.23 \pm \\
0.12\end{array}$ \\
\hline $\mathrm{d}$ & $\begin{array}{l}2.91 \pm \\
0.34\end{array}$ & $\begin{array}{l}8.18 \pm \\
0.27\end{array}$ & $\begin{array}{c}28.77 \pm \\
0.62\end{array}$ & $\begin{array}{c}33.06 \pm \\
0.65\end{array}$ & 98.8 & 28.1 & 28.4 & $\begin{array}{c}32.36 \pm \\
0.88\end{array}$ & $\begin{array}{c}35.21 \pm \\
0.58\end{array}$ & $\begin{array}{c}25.61 \pm \\
0.13\end{array}$ & $\begin{array}{c}23.83 \pm \\
0.18\end{array}$ & $\begin{array}{c}9.73 \pm \\
0.14\end{array}$ & $\begin{array}{l}1.26 \pm \\
0.09\end{array}$ \\
\hline $\mathrm{e}$ & $\begin{array}{c}2.96 \pm \\
0.42\end{array}$ & $\begin{array}{c}8.62 \pm \\
0.89\end{array}$ & $\begin{array}{c}28.98 \pm \\
0.73\end{array}$ & $\begin{array}{c}31.97 \pm \\
0.72\end{array}$ & 97.9 & 29.1 & 29 & $\begin{array}{c}30.94 \pm \\
0.93\end{array}$ & $\begin{array}{c}31.91 \pm \\
0.18\end{array}$ & $\begin{array}{c}22.7 \pm \\
0.25\end{array}$ & $\begin{array}{c}22.14 \pm \\
0.21\end{array}$ & $\begin{array}{c}9.73 \pm \\
0.14\end{array}$ & $\begin{array}{c}1.13 \pm \\
0.21\end{array}$ \\
\hline
\end{tabular}

* Low significant $\mathrm{P}=<0.05$

** Moderate significant $\mathrm{P}<0.01$

Table 4: Average body weight (ABW), weight gain (WG), feed consumption (FC), feed conversion rate (FCR) in the different experimental groups.

\begin{tabular}{|c|c|c|c|c|c|c|c|c|c|c|c|c|c|}
\hline \multirow[t]{2}{*}{ Parameters } & \multirow{2}{*}{$\begin{array}{l}\text { A.B.W. at } \\
20 \text { days }\end{array}$} & \multicolumn{4}{|c|}{27 days } & \multicolumn{4}{|c|}{34 days } & \multicolumn{4}{|c|}{41 days } \\
\hline & & A.B.W. & W.G & F.C. & F.C.R & A.B.W. & W.G & F.C. & F.C.R. & A.B.W. & W.G & F.C. & F.C.R. \\
\hline Non infected non treated & $\begin{array}{c}574.21 \\
\pm \\
3.21\end{array}$ & $\begin{array}{c}874.28 \\
\pm \\
5.28\end{array}$ & $\begin{array}{c}300.07 \\
\pm \\
4.29\end{array}$ & 680.21 & 2.27 & $\begin{array}{c}1323.26 \\
\pm \\
5.27\end{array}$ & $\begin{array}{c}448.98 \\
\pm \\
2.51\end{array}$ & 900.24 & 2.005 & $\begin{array}{c}1805.18 \\
\pm \\
4.38\end{array}$ & $\begin{array}{c}481.92 \\
\pm \\
2.53\end{array}$ & 940.46 & 195 \\
\hline Infected non treated & $\begin{array}{c}573.24 \\
\pm \\
4.32\end{array}$ & $\begin{array}{c}853.15 \\
\pm \\
6.25^{*}\end{array}$ & $\begin{array}{c}279.91 \\
\pm \\
4.23 *\end{array}$ & 664.35 & 2.38 & $\begin{array}{c}1295.96 \\
\pm \\
3.34 * *\end{array}$ & $\begin{array}{c}442.81 \\
\pm \\
2.27 *\end{array}$ & 899.48 & 2.03 & $\begin{array}{c}1766.04 \\
\pm \\
6.36 * *\end{array}$ & $\begin{array}{c}470.08 \\
\pm \\
2.09^{*}\end{array}$ & 928.57 & 1.98 \\
\hline $\begin{array}{l}\text { Infected treated with } \\
\text { (tol) }\end{array}$ & $\begin{array}{c}579.51 \\
\pm \\
4.42\end{array}$ & $\begin{array}{c}894.38 \\
\pm \\
3.27 * *\end{array}$ & $\begin{array}{c}314.87 \\
\pm \\
3.29 *\end{array}$ & 684.07 & 2.17 & $\begin{array}{c}1346.18 \\
\pm \\
5.37 *\end{array}$ & $\begin{array}{c}451.8 \\
\pm \\
4.31\end{array}$ & 912.09 & 2.01 & $\begin{array}{c}1833.27 \\
\pm \\
5.27\end{array}$ & $\begin{array}{c}487.0 \\
\pm \\
2.45 *\end{array}$ & 942.48 & 1.94 \\
\hline $\begin{array}{l}\text { Infected treated with } \\
\text { (amp) }\end{array}$ & $\begin{array}{c}576.36 \\
\pm \\
3.72\end{array}$ & $\begin{array}{c}890.92 \\
\pm \\
6.37 *\end{array}$ & $\begin{array}{c}314.56 \\
\pm \\
3.48 *\end{array}$ & 682.19 & 2.17 & $\begin{array}{c}345.46 \\
\pm \\
4.39 *\end{array}$ & $\begin{array}{c}454.54 \\
\pm \\
4.29\end{array}$ & 920.39 & 2.02 & $\begin{array}{c}1831.38 \\
\pm \\
5.51\end{array}$ & $\begin{array}{c}485.29 \\
\pm \\
2.09^{*}\end{array}$ & 942.93 & 1.94 \\
\hline $\begin{array}{l}\text { Infected treated with (tol } \\
\pm \text { amp) }\end{array}$ & $\begin{array}{c}579.43 \\
\pm \\
4.398\end{array}$ & $\begin{array}{c}897.27 \\
\pm \\
4.19^{* *}\end{array}$ & $\begin{array}{c}317.87 \\
\pm \frac{7}{4} *\end{array}$ & 687.59 & 2.16 & $\begin{array}{c}1355.26 \\
\pm \\
3.57 * *\end{array}$ & $\begin{array}{c}457.99 \\
\pm \\
3.57\end{array}$ & 922.52 & 2.01 & $\begin{array}{c}1846.59 \\
\pm \\
4.43\end{array}$ & $\begin{array}{c}491.33 \\
\pm \\
1.64\end{array}$ & 941.38 & 1.92 \\
\hline
\end{tabular}

* Low significant $\mathrm{P}=<0.05$

** Moderate significant $\mathrm{P}<0.01$ 
Table 5: Shows mean HI, results of challenge with VVNDV, bursal weight, body weight, bursal body weight ratio and bursal body weight index in the different experimental groups at 40 day of age.

\begin{tabular}{|c|c|c|c|c|c|c|}
\hline $\begin{array}{c}\text { 苛 } \\
\text { Mean HI titer }\end{array}$ & $\begin{array}{c}\text { Protection \% post } \\
\text { VVNDV challenge }\end{array}$ & $\begin{array}{c}\text { Mean bursal } \\
\text { weight }\end{array}$ & $\begin{array}{c}\text { Mean body } \\
\text { weight }\end{array}$ & $\begin{array}{c}\text { Bursal body } \\
\text { weight ratio }\end{array}$ & $\begin{array}{c}\text { Bural body } \\
\text { weight index }\end{array}$ \\
\hline $\mathrm{a}$ & $2.947 \pm 0.4$ & $90 \%$ & $1.9 \pm 0.5$ & $1805 \pm 4.38$ & 0.00105 & \\
\hline $\mathrm{b}$ & $2.5 \pm 0.3$ & $70 \%$ & $2.1 \pm 0.2$ & $1766 \pm 6.36^{* *}$ & 0.0011 & 1.132 \\
\hline $\mathrm{c}$ & $2.9 \pm 0.29$ & $90 \%$ & $1.9 \pm 0.4$ & $1833 \pm 5.27$ & 0.00103 & 0.95 \\
\hline $\mathrm{d}$ & $3.1 \pm 0.4$ & $90 \%$ & $2.1 \pm 0.3$ & $1831 \pm 5.51$ & 0.00114 & 1.08 \\
\hline $\mathrm{e}$ & $\begin{array}{c}3.0 \pm 0.38 \\
\text { n.s. }\end{array}$ & $90 \%$ & $\begin{array}{c}2.3 \pm 0.2 \\
\text { n.s. }\end{array}$ & $1846 \pm 4.43$ & $\begin{array}{c}0.00124 \\
\text { n.s }\end{array}$ & 1.186 \\
\hline
\end{tabular}

$* * \mathrm{P}<0.01$

\section{DISCUSSION}

Weight gain and coccidial lesion score are primary criteria for measuring the anticoccidial efficacy Conway et al. (1990). In the present study clinical signs, mortality rate, lesion score, oocyst count, performance and hematological picture was performed, beside the liver and kidney functions and some immunological criteria, to examine the safe usage of the tested drugs.

Clinically the positive control group (b) showed reduced feed intake, depression, ruffled feather, debility, dropped head, blood diarrhea, $10 \%$ mortality, the highest lesion score and the highest oocyst count post infection (PI). (Table 2). These findings are in agreement with the previous findings of Ahmed (2004) and Eid et al. (2004).

The hemogram of negative control group (a) RBCs count, HB\%, PCV and WBCs count were similar to those reported by Twisselmann (1939); Pilaski (1972); Lucas and Jamaroz (1961) and Olson (1937) respectively. RBCs count, $\mathrm{HB} \%$, PCV values were significantly lower in group (b) (Table 3). this is due to hemorrhagic enteritis due to coccidial infection (Conwary et al., 1993; McDogald, 2003; Eid et al., 2004; Seddik and El-Bealawy 2007), while the significant increase in the total leucocytic count may be due to enteritis specially if we consider that this group was showing the highest enteric lesion score (Table 2). Similarly found Seddik and El-Bealawy (2007). 
Aspartate aminotransferase (AST) and alanine aminotransferase (ALT) are transaminases enzymes, they had great clinical importance in the inter conversion of aminoacid and oxoacid by transfer of amino group. ALT increases due to pathologic changes in all tissues. AST activity greater than $23 \mathrm{u} / \mathrm{L}$ are considered abnormal and it reflects repatic, skeletal muscles or kidney changes. Alkaline phosphatase (ALP) in involved in energy transfer for exchange of ions across the cell membrane, this activity is present in liver, intestine and bone. Elevation of its level indicates cellular damage (Lumeij, 1988; El-Sayed 2002). Gamma glutamy transferase (GGT) cleaves the Gamma glutamyl group from peptides and moves them to an appropriate acceptor, these enzymes are of great importance in biliary and renal tubular epithelium. (GGT) activity in the serum is from biliary origin (Hochleithner, 1994). (AST), (ALT), (ALP) and (GGT) could be used as indicative for hepatic pathology (Lewandowoki et al., 1986).

In the present study negative control group (a) showed the lowest value for (AST), (ALT) (ALP) and (GGT) (Table 3). Positive control group (b) showed moderate significant increase in AST, ALT, ALP and GGT (Table 3). Similar to the previous findings of Fukata et al., (1997); Eid et al. (2004) and Sameh et al. (2005) this could be attributed to the dehydration resulting from diarrhea caused by coccidia Mc Dougold (2003) group (c,d) receiving toltazuril or amprolium plus individually showed high non significant ALT, AST, ALP and GGT compared to negative control meanwhile group (e) receiving toltrazuril and amprolium plus simultaneously had a non significant low ALT, AST, ALP and GGT as compared with the negative control group or the individually treated groups pointing to the value of concurrent use of the two drugs (Table 3).

Uric acid is the primary catalatic product of protein and non protein nitrogenous in birds, it is excreted by the avian kidney primarily by tubular excretion, therefore elevated serum uric acid values are expected in birds with impaired renal function and reduced renal uric acid clearance. The normal blood uric acid values for most birds ranges between $2-10 \mathrm{mg} / \mathrm{dl}$.

Creatinine is not a major non protein nitrogen component in avian blood, it has a questionable value in evaluating renal function in birds, its normal value in most birds is $0.2 \mathrm{mg} / \mathrm{dl}$. Creatinine well be elevated slightly in renal failure $0.5-1.5 \mathrm{mg} / \mathrm{dl}$ but is less reliable than 
uric acid in evaluating renal functions, because birds excretes creatine in their urine before it has been converted to creatinine. The measurement obtained as normal for creatinine may be pseudocreatinine such as glucose, protein, ascorbic acid and pyrivic acid thus it may not reflect glomerular function.

In the present study groups ( $a, b, c, d$ and e) had serum uric acid values ranging from 8.03 up to 10.73 within the high normal physiologic limits (Table 3) and the creatinine values were ranging from 1.02 up to $1.48 \mathrm{mg} / \mathrm{dl}$, the high values in the non treated group (a) lead us to consider the high protein level of the consumed diet (high energy - high protein), to be the cause for this high measurement of kidney function parameter (Lewandowski et al., 1986).

Treatment of infected chickens with the therapeutic dose of toltrazuril or amprolium plus reduced the clinical signs and improved the health status of infected groups as evidenced by the decreased mortality rate, lesion score and oocyst output. Similar results for amprolium were obtained by Tocchini and Tassi (1984); Chapman (1989); Chapman (1999) and Hernandez et al. (2000) while similar results for toltrazuril treatment was obtained by Khaled (1994); Ramadan et al. (1997) and Sameh et al. (2005).

The reduction in oocyst output following treatment with amprolium is due to its effect on the first generation schizont Danforth and Anderson (1989); Vertommen and Peak (1990) stated that the efficacy of toltrazuril is due to its effect on sexual stages of coccidia and also through the inhibition of nuclear division of schizont.

Performance parameters such as body weight, weight gain, feed consumption, feed conversion rate in experimental groups treated with toltrazuril or amprolium plus were improved when compared to the non infected non treated group. Similarly found Sameh et al. (2005) and Mohamed (2008) the improvement of performance parameters may be due to improvement of general health conditions, increase food intake and absorption of nutrients as mentioned by Abdein and Abd El-Fattah (2003), but Joyner et al. (1963) stated that this effect would be due to improvement of the integrity of the epithelial lining of the gastro intestinal tract.

In the present study B:B index, challenge results, and serology were used to evaluate immunosuppression after IBDV vaccination or toltrazuril and amprolium medication. B:B index for the different 
experimental groups were ranging from (0.95-1.186). Lucio and Hitchner (1979) found that B:B index lesser than (0.7) signifies immunosuppresion this was not encountered in any of the experimental groups.

The protection $\%$ against VVNDV was $70 \%$ in group (B) this may be due to disease concurrency post coccidial infection as mentioned by Mc Doughald and Reid (1991), in the remaining groups the protection \% was (90\%). This is correlated with the $\mathrm{HI}$ immune titer as mentioned by Allan et al. (1978).

From the above mentioned data we could conclude that the tested drugs have no effect on the immune competance this is evident from the results of $\mathrm{B}: \mathrm{B}$ index, serology, and challenge in the different tested groups.

\section{REFERENCES}

Abdein, H.M. and Abd El-Fattah, M. (2003): Comparative study on some anticoccidial programes in broilers. Zag. Vet. J. 31: 7996.

Ahmed, H.E. (2004): Possible antiparasitic activities of ambrosia maritime in experimental animals. MVSc. Fac. of Vet. Med. Zag. Univ.

Alexander, J.T. (2002): Coccidosis in poultry disease $5^{\text {th }}$ ed, pp. 405420 edited by Jordan FTW, pattison M., Alexander D, farager T.W.B. sauaders London, Edinburgh. New York Philadelphia. St Loius Sydney Toronto.

Allan, W.H.; Lancaster, J.E. and Toth, B. (1978): Newcastle disease vaccines: their production and use. FAO Animal Production and health series No. 10 Rome.

Bayoumie, H.A.A. (1997): Immune response in chickens after simultaneous vaccination against Newcastle and Gumboro diseases viruses. MVSc. Thesis Poult. Dis. Fac. of Vet. Med., Zag. Univ.

Bayoumie, H.A.A.; EL-Sakkar, G.H. and Allam, H.H. (2006): Viral inducing proventriculitis casues and pathological findings in chickens. $8^{\text {th }}$ Sci. Vet. Med. Zag. Conference 31 Aug. -3 Sept. 2006 Harghada pp. 564-576. 
Caraway, W. (1957): Calorimetric determination of uric acid with deproteinzation. Am. J. Clin. Path. 25: 840.

Chapman, H. (1989): Chemotherapy of caecal coccidiosis: efficacy of toltazuril sulphaquinoxaline pyrimethamine and amprolium / ethopabate given in drinking water against field isolates of $\mathrm{E}$. tenella. Res. Vet. Sci. 46: 419-420.

Chapman, H. (1999): Anticoccidial drug and their effects upon the development of immunity to Eimeria infection in poultry. Avian Path. 28: 521-535.

Coles, E. (1986): Veterinary clinical pathology $4^{\text {th }}$, Ed. WB saunders pheladelphia USA.

Conway, D.; McKenzie, M. and Dayton, A. (1990): Relationship of coccidial lesion scores and weight gain in infections of $\mathrm{E}$. acervulina, E. maxima and E. tenella in broilers. Avian Path. 19: 489-496.

Conway, D.; Sassai, K.; Gaafar, S. and Smothrs, C. (1993): Effect of different levels of oocyst inocula of E. acerulina, E.tenella and E. maxima on plasma constituents, packed cell volume lesion score and performance in chickens. Avian Dis. 37: 48-123.

Danforth, H. and Anderson, W. (1989): The ultrastructural effect of amprol plus medication on development of immunity to Eimeria infection in poultry. Avian Dis. 33: 303-309.

Eid, A.A.; Abd El-Lateif, A.E.; EL-Dieb, M.M. and Said, M.A. (2004): Anticoccidial efficiency of Artemisiacina and its extracts in broiler chickens. Zag. Vet. J. 32: 1-14.

El-Sayed, N.A. (2002): Effect of recent anticoccidial drugs on chickens. MVSc. Thesis, Pharmacology Dept. Fac. of Vet. Med., Zag. Univ.

Fukata, T.; Komba, Y.; Sasai, K. and Arakawa, A. (1997): Chemistry and haematological studies on chickens infected with Eimeria tenella and E. acervulina. Vet. Rec. 141: 44-46.

Hernandez, M.; Zezypel, B. and Larramendy, R. (2000): Evaluation of salinomyein and amprolium plus against avian coccidiosis in the laboratory. Revista cubana de- ciencia. Avicola 24: 65-67.

Hochleithner, M. (1994): Biochemistries in avian medicine principles and application edited by richie B, Harrison G, and Harrison L. Wingers Publishing Co. 
Husdan, H. and Rapaport, A. (1968): Chemical determination of creatine with depolarization. Clin. Chem. 14: 222-228.

Johnson, J. and Reid, W. (1970): Anticocidal drugs: lesion scoring technique in battery and floor pen experiments with chickens. Experimental parasit. 28: 30-33.

Jordan, F.T.W. (1990): Coccidiosis in poultry diseases $3^{\text {rd }}$ ed. Pp. 226241. ELBS. English language book society / bailliere Tindall.

Jordan, F.T.W. and Pattison, M. (1996): Coccidiosis in poultry diseases $4^{\text {th }}$ ed. Pp. 261-276. W. B. Saunders company ltd. London Philadelphia . Toronto. Sydney Tokyo.

Joyner, L.P.; Davies, S.F.M. and Kendall, S.B. (1963): Chemotherapy of coccidosis in experimental chemotherapy Vol. 1 (Eds) Schnitzer R.J. \& Hawking F. (PP. 445-486) London, Academic Press.

Karim, M. and Tress, A. (1990): Isolation of five species of eimeria in Bangladesh. Tropical Anim. Health Prod. 22: 153-159.

Khaled, K.A. (1994): Study on the interaction of some anticoccidial drugs. MVSc. Thesis Fac. of Vet. Med., Zag. Univ.

Lewandowki, A.H.; Campbell, T.W. and Harrison, G.J. (1986): Clinical chemistries in clinical avian medicine and surgery edited by Harrison, G.J. and Harrison, L R. Sannders W, B. Company.

Lucas, A.M. and Jamaroz, C. (1961): Atlas of avian hematology US. Dept. of agriculture monograph 25.

Lucio, B. and Hitchner, S.B. (1979): Infectious bursal disease emulsified vaccine effect upon neutralizing antibody level in the dam and subsequent protection of the progeny. Avian Dis. 23: 466-478.

Lumeij, J.T. (1988): Avian clinical pathology some experimental findings of importance to the practitioner. Proc. Assoc. Avian Vet. Pp. 79-86.

McDougald, L.R. (2003): Coccidiosis in diseases of poultry $11^{\text {th }}$ Ed. Pp. 974-991. Edited by Saif Y. M. Iowa State Press. A black well Publishing Company.

McDougald, L.R. and Reid, W.M. (1991): Coccidiosis in diseases of poultry edited by calnek BW, Barnes HJ, Beared CW, Reid WM and Yodor H.W. Jr. Iowa State University Press. Ames. Iowa, USA. 
Mohamed, H.M. (2008): Studies on the interaction of some chemicals on the control of chicken diseases. MVSc. Thesis Fac. of Vet. Med., Suez Canal Univ.

Olson, C. (1937): Variation in cells and hemoglobin content in blood of normal domestic chicken. Cornell Vet. 27: 235.

Pilaski, J. (1972): Vergleichende untersuchungen uber den hamoglobingehalt des huhner und putenblutes in abbargigkeir vor attar und geschlecht. Arch. Gefflugelkunde 36: 70.

Ramadan, A.; Abo El-Sooud, K. and El-Baly, M. (1997): Anticoccidial efficacy of toltrazuril and halfungione against E. tenella infection in broiler chickens in Egypt. Res. Vet. Sc. 62: 175178.

Reitman, S. and Franckel, S. (1957): A colorimetric determination of serum AST and ALT isoenzymes. Am. J. Clin. Path. 28: 56-58.

Sameh, M.; Nasser, A. and Gehan, N.G. (2005): Effect of secnidazole on Eimeria Tenella. $4^{\text {th }}$ Int. Sci. Conf. Fac. of Vet. Med. Mansoura Univ.: 1411-1428.

Seddik, A. and El-Bealawy, M. (2007): The effect of probiotic in broiler chickens infected with E. maxima. Zag. Vet. J. 35: 189-201.

Snedecor, G.W. and Cochran, W.S. (1967): Statistical methods $6^{\text {th }}$ ed., Iowa Univ. Press, Ames. Iowa, USA.

Szaz, G. (1969): Quantitative determination of gamma glutamyle transferase in serum or plasma. Clin. Chem. 22: 124-136.

Tietz, N.W. (1986): Textbook of clinical chemistry. Philadelphia, WB Sanders Co.

Tocchini, M. and Tassi, P. (1984): Efficacy and effect on productivity of anticoccidial used alternately in broilers. Clin. Vet. 107: 297310 .

Twisselman, N.M. (1939): A study of the cell content of blood of normal chickens with supravital stains. Poult. Sci. 18: 151.

Vertommen, M. and Peak, H. (1990): Efficacy of totrazuril in broilers and development of laboratory model for sensitivity testing of eimeria field isolates. Vet. Quarterly, 12: 183-192.

Villegas, P. (1991): Laboratory manual for avian virus diseases (AM 80 S). The University of Georgia, College of Vet. Med. Athens, Georgia. 30602. 